\title{
Haplotype-based heritability estimations reveal gestational duration as a maternal trait and fetal size measurements at birth as fetal traits in human pregnancy
}

Amit K. Srivastava ${ }^{1}$, Julius Juodakis ${ }^{2}$, Pol Sole-Navais ${ }^{2}$, Jing Chen ${ }^{3}$, Jonas Bacelis ${ }^{2,4}$, Kari Teramo $^{5}$, Mikko Hallman ${ }^{6}$, Pal R. Njølstad ${ }^{7}$, David M. Evans ${ }^{8}$, Bo Jacobsson ${ }^{9,10}$, Louis J. Muglia ${ }^{1}$, Ge Zhang ${ }^{1 *}$

\section{Affiliations}

1) Division of Human Genetics, Cincinnati Children's Hospital Medical Center, USA; The Center for Prevention of Preterm Birth, Perinatal Institute, Cincinnati Children's Hospital Medical Center, USA; March of Dimes Prematurity Research Center Ohio Collaborative, USA; Department of Pediatrics, University of Cincinnati College of Medicine, USA; 2) Department of Obstetrics and Gynecology, Institute of Clinical Sciences, Sahlgrenska Academy, University of Gothenburg, Gothenburg, Sweden; 3) Division of Biomedical Informatics, Cincinnati Children's Hospital Medical Center, Cincinnati, OH, USA; 4) Region Västra Götaland, Sahlgrenska University Hospital, Department of Obstetrics and Gynecology, Gothenburg, Sweden; 5) Obstetrics and Gynecology, University of Helsinki and Helsinki University Hospital, Helsinki, Finland; 6) PEDEGO Research Unit and Medical Research Center Oulu, University of Oulu and Department of Children and Adolescents, Oulu University Hospital, Oulu, Finland; 7) KG Jebsen Center for Diabetes Research, Department of Clinical Science, University of Bergen, Bergen, Norway; Division of Health Data and Digitalization, Department of Genetics and Bioinformatics, Norwegian Institute of Public Health, Oslo, Norway; Center for Medical Genetics and Molecular Medicine, Haukeland University Hospital, Bergen, Norway; 8) University of Queensland Diamantina Institute, University of Queensland, Brisbane, QLD, Australia; Medical Research Council Integrative Epidemiology Unit, University of Bristol, Bristol, BS8 2BN, UK.; Bristol Medical School, Population Health Sciences, University of Bristol, Bristol, UK.; 9) Department of Obstetrics and Gynecology, Sahlgrenska Academy, University of Gothenburg, Gothenburg, Sweden.; 10) Department of Genetics and Bioinformatics, Area of Health Data and Digitalization, Norwegian Institute of Public Health, Oslo, Norway

*Correspondence: Ge.Zhang@cchmc.org 


\begin{abstract}
Unlike other commonly studied complex traits, pregnancy phenotypes like gestational duration and fetal size measurements at birth are conjointly determined by maternal and fetal genomes. Current approaches of heritability estimation based upon an individual's genotype information are limited in addressing confounding by shared alleles between mother and fetus. Here, we propose a novel approach of treating the mother-child pairs as a single analytical unit with three haplotypes - maternal transmitted (m1), maternal non-transmitted (m2) and paternal transmitted (p1). Using our haplotype-based approach, we estimate the SNP heritability $\left(\hat{\mathrm{h}}^{2}\right)$ of gestational duration and gestational duration adjusted fetal size measurements at birth in 10,375 mother-child pairs. The results reveal that variance in gestational duration is mainly attributable to $\mathrm{m} 1$ and $\mathrm{m} 2$ ( $\hat{\mathrm{h}}_{\mathrm{m} 1}^{2}=$ $14 \%$ and $\hat{\mathrm{h}}_{\mathrm{m} 2}^{2}=10 \%$ ). In contrast, variance in fetal size measurements at birth are mainly attributable to $\mathrm{m} 1$ and $\mathrm{p} 1$. Variance in birth weight is attributable to both $\mathrm{m} 1$ and $\mathrm{p} 1$ ( $\hat{\mathrm{h}}_{\mathrm{m} 1}^{2}=$ $19.9 \%$ and $\left.\hat{\mathrm{h}}_{\mathrm{p} 1}^{2}=13.3 \%\right)$. However, variance in birth length $\left(\hat{\mathrm{h}}_{\mathrm{m} 1}^{2}=24.5 \%\right.$ and $\left.\hat{\mathrm{h}}_{\mathrm{p} 1}^{2}=4.0 \%\right)$ and head circumference $\left(\hat{\mathrm{h}}_{\mathrm{m} 1}^{2}=33.1 \%\right.$ and $\left.\hat{\mathrm{h}}_{\mathrm{p} 1}^{2}=12.3 \%\right)$ are largely attributable to $\mathrm{m} 1$. Our results suggest that gestational duration is primarily determined by the maternal genome whereas fetal size measurements at birth are primarily determined by fetal genome. In addition, the difference between $\left(\hat{\mathrm{h}}_{\mathrm{m} 1}^{2}-\widehat{\mathrm{h}}_{\mathrm{m} 2}^{2}\right)$ and $\hat{\mathrm{h}}_{\mathrm{p} 1}^{2}$ suggests a greater contribution of the maternal transmitted haplotype than the paternal transmitted haplotype to birth length and head circumference. Our haplotype-based GCTA approach (H-GCTA) resolves explicit contributions of maternal and fetal genomes to SNP heritability of pregnancy phenotypes.
\end{abstract}

Keywords: Narrow-sense heritability, SNP heritability, pregnancy phenotypes, transmitted and non-transmitted alleles 


\section{Introduction}

Narrow sense heritability $\left(\mathrm{h}^{2}\right)$ is the proportion of phenotypic variance in a population attributable to additive genetic values (breeding values) ${ }^{1}$. Generally, the concept of the $\mathrm{h}^{2}$ estimation comes from balanced designs - regression of child phenotypes on mid-parent phenotype, correlation of full or half sibs and differences in the correlation of monozygotic and dizygotic twins ${ }^{1}$. However, in a population with mixed relationships, the linear mixed model (LMM) is the most flexible approach accounting for both fixed and random effects ${ }^{1-5}$. LMM is generally fitted using restricted maximum likelihood (REML) ${ }^{5-7}$, Expectation Maximization (EM) ${ }^{8}$ or Bayesian methods ${ }^{9,10}$. Following the advent of genome-wide common SNPs arrays, imputation and whole genome sequencing technologies encouraged researchers to extend its applicability to distantly related and unrelated individuals.

Over the last decade, various approaches including Genome-based Restricted Maximum Likelihood (GREML) ${ }^{11,12}$, Linkage Disequilibrium-adjusted kinships (LDAK) ${ }^{13}$, threshold Genomic Relatedness Matrices (GRMs) ${ }^{14}$, LD Score regression (LDSC) ${ }^{15}$ and Phenotype Correlation-Genotype Correlation (PCGC) ${ }^{16}$ have been developed to estimate SNP-based narrowsense heritability $\left(\hat{\mathrm{h}}^{2}\right)^{17}$. In addition, variants of these approaches such as GREML-MAF stratified $\left(\right.$ GREML-MS) ${ }^{18}$, GREML-LD and MAF stratified (GREML-LDMS) ${ }^{19}$ and LDAK-MAF stratified $\left(\right.$ LDAK-MS ${ }^{20}$ have enabled partitioning of the genetic variance into additive and non-additive components as well as variance components attributable to chromosomes, genes and inter-genic regions. The above approaches have helped explain a large proportion of the missing heritability in various complex diseases and quantitative traits. Nevertheless, they are less suited for pregnancy phenotypes which are simultaneously influenced by direct fetal genetic effects and indirect parental effects ${ }^{21-24}$. To date, only a few studies have attempted to distinguish maternal genetic effect ${ }^{25,26}$ from fetal genetic effect in mother-child duos ${ }^{21,23,27,28}$. Most of these approaches are still based on individuals' genotypes and therefore, are inadequate in addressing the confounding effect of shared alleles between mother and fetus. Hence, pregnancy-related, antenatal and perinatal phenotypes demand an approach which can avoid this confounding and partition $\hat{\mathrm{h}}^{2}$ explicitly into maternal and fetal components.

Here, we introduce a haplotype-based genome-wide complex trait analysis approach (H-GCTA) for heritability estimation to resolve explicit contribution of maternal and fetal genomes to the variance of pregnancy outcomes. We consider mother-child pairs as single analytical units consisting of three haplotypes corresponding to maternal transmitted (m1), maternal nontransmitted (m2) and paternal transmitted (p1) alleles ${ }^{29,30}$. Use of such an analytical unit provides an advantage over conventional approaches based on individual's genotype information by avoiding the confounding of $\mathrm{m} 1$ which can influence pregnancy phenotypes through both the mother and fetus (Fig 1a) ${ }^{24}$. We generate three separate genetic relatedness matrices M1, M2 and $\mathrm{P} 1$ using only $\mathrm{m} 1$, only $\mathrm{m} 2$ and only $\mathrm{p} 1$ respectively. We fit all three matrices simultaneously in a linear mixed model (LMM) and Haseman-Elston (HE) regression ${ }^{31-33}$ to estimate variance components attributable to each of the haplotype-based matrices (Fig 1b). We apply our approach 
to a cohort of 10,375 mother-child pairs to estimate $\hat{\mathrm{h}}^{2}$ of gestational duration and gestational duration adjusted fetal size measurements at birth e.g. birth weight, birth length and head circumference. Our results suggest that genetic variance in gestational duration is primarily attributable to the maternal genome i.e. the maternal transmitted (m1) and non-transmitted (m2) alleles whereas genetic variance in fetal size measurements at birth are mainly attributable to fetal genome - maternal transmitted (m1) and paternal transmitted (p1) alleles. We additionally reveal a larger contribution of the maternal transmitted haplotype than the paternal transmitted haplotype $\left(\hat{\mathrm{h}}_{\mathrm{m} 1}^{2}-\widehat{\mathrm{h}}_{\mathrm{m} 2}^{2}>\widehat{\mathrm{h}}_{\mathrm{p} 1}^{2}\right)$ for birth length and head circumference. Our approach can not only estimate explicit maternal and fetal contribution to pregnancy phenotypes but also detect parent-of-origin effects (POEs) or correlation between maternal and fetal genetic effects of the maternal transmitted alleles. 


\section{Results}

We first used simulated data to evaluate the validity of H-GCTA and its capabilities in detecting POEs. We applied our approach to estimate SNP-based narrow-sense heritability $\left(\hat{\mathrm{h}}^{2}\right)$ of four pregnancy phenotypes - gestational duration, birth weight, birth length and head circumference in 10,375 mother-child pairs. The study cohorts included five European cohorts - Avon Longitudinal Study of Parents and Children (ALSPAC) ${ }^{34,35}$, Danish Birth Cohort (DNBC) ${ }^{36}$, Norwegian Mother, Father and Child Cohort study $(\mathrm{MoBa})^{37}$, Hyperglycemia and Adverse Pregnancy Outcome study (HAPO) ${ }^{38}$ and Finnish dataset (FIN) ${ }^{30,39}$ (Supplementary Text and Supplementary Tables 1-4). We compared results from our approach with two existing approaches namely, GCTA $^{11}$ and M-GCTA ${ }^{23,28}$ (Supplementary Figure 1). Further, $\hat{\mathrm{h}}^{2}$ estimation of gestational duration was replicated in mother-child pairs from another Norwegian dataset (HARVEST) (Supplementary Text).

\section{Heritability Estimation using simulated data}

We used simulated genotype and phenotype data to assess the utility and robustness of our approach (H-GCTA). We estimated $\hat{\mathrm{h}}^{2}$ in 3,000 trios using 10,000 SNPs with MAF > 0.001. All phenotypes were simulated with 50\% SNP-based narrow sense heritability $\left(\hat{\mathrm{h}}^{2}=0.5\right)$. Phenotypes were simulated in three ways - maternal phenotypes i.e. only maternal transmitted and nontransmitted alleles affecting phenotype, paternal phenotypes i.e. only paternal transmitted and nontransmitted alleles affecting phenotype and fetal phenotypes i.e. only maternal transmitted and paternal transmitted alleles affecting phenotype. For fetal traits, we also incorporated different levels of POE (see methods).

\section{Heritability of simulated maternal and paternal traits}

Using conventional GCTA for maternal traits in mothers, fathers and children separately, the estimated $\hat{\mathrm{h}}^{2}$ based on maternal (m), paternal (p) and fetal (f) genotypes was $49.8 \%, 2.2 \%$ and $12.0 \%$ respectively (Supplementary Table 5). We also used M-GCTA in mother-child duos to estimate the phenotypic variance of maternal traits attributable to indirect maternal effect $\left(\hat{\mathrm{h}}_{\mathrm{M}^{\prime}}^{2}=\right.$ $48.4 \%)$, direct fetal effect $\left(\hat{\mathrm{h}}_{\mathrm{G}}^{2}=1.5 \%\right)$ and direct-indirect effect covariance $\left(\hat{\mathrm{h}}_{\mathrm{D}}^{2}=1.3 \%\right)$ (Supplementary Table 5). Using H-GCTA for maternal traits in complete trio data, phenotypic variance based on maternal transmitted alleles $\left(\hat{\mathrm{h}}_{\mathrm{m} 1}^{2}\right)$, maternal non-transmitted alleles $\left(\hat{\mathrm{h}}_{\mathrm{m} 2}^{2}\right)$, paternal transmitted alleles $\left(\hat{\mathrm{h}}_{\mathrm{p} 1}^{2}\right)$ and paternal non-transmitted alleles $\left(\hat{\mathrm{h}}_{\mathrm{p} 2}^{2}\right)$ was $23.0 \%, 23.3 \%$, $1.6 \%$ and $1.5 \%$ respectively (Fig 2a, Supplementary Table 5). M-GCTA and H-GCTA accurately distinguished the maternal origin of the simulated phenotype; however, the conventional GCTA also showed a superficial contribution from the fetal genome $(12.0 \%$, approximately one quarter of the $\hat{\mathrm{h}}^{2}$ based on maternal genotype) due to allele transmission. As expected, $\hat{\mathrm{h}}^{2}$ estimates for paternal traits followed similar patterns as $\hat{\mathrm{h}}^{2}$ estimates for maternal traits (Supplementary Table 5).

\section{Heritability of simulated fetal traits without POEs}


Like parental traits, we used conventional GCTA to estimate $\hat{\mathrm{h}}^{2}$ for fetal traits in mothers, fathers and children separately. The estimated $\hat{\mathrm{h}}^{2}$ based on $\mathrm{m}, \mathrm{p}$ and $\mathrm{f}$ were $12.7 \%, 13.5 \%$ and $49.6 \%$ respectively (Supplementary Table 5). Similarly, using M-GCTA for fetal traits in mother-child duos, the $\widehat{h}^{2}$ estimates attributable to indirect maternal effect (M'), direct fetal effect $(G)$ and directindirect effect covariance (D) were $2.0 \%, 48.2 \%$ and $1.4 \%$ respectively (Supplementary Table 5). Using H-GCTA in complete trio data, we further resolved the phenotypic variance of the simulated fetal traits into variance components attributable to $\mathrm{m} 1\left(\hat{\mathrm{h}}_{\mathrm{m} 1}^{2}=23.2 \%\right), \mathrm{m} 2\left(\hat{\mathrm{h}}_{\mathrm{m} 2}^{2}=1.5 \%\right), \mathrm{p} 1$ $\left(\hat{\mathrm{h}}_{\mathrm{p} 1}^{2}=24.1 \%\right)$ and $\mathrm{p} 2\left(\hat{\mathrm{h}}_{\mathrm{p} 2}^{2}=1.2 \%\right)($ Fig $2 \mathrm{~b}$, Supplementary Table 5). While, conventional GCTA estimated superficial contributions from maternal and paternal genotypes besides fetal genotypes, M-GCTA and H-GCTA clearly showed the fetal origin of the simulated phenotypes. As compared to M-GCTA, H-GCTA further resolved equal contributions from maternal and paternal transmitted alleles through $\mathrm{m} 1$ and $\mathrm{p} 1$.

\section{Heritability of simulated fetal traits with POEs}

We estimated variance attributable to POEs using simulated fetal traits where $\mathrm{m} 1$ had less effect in comparison to $\mathrm{p} 1$. We simulated four scenarios where different fractions of causal variants $(25 \%, 50 \%, 75 \%$ or $100 \%)$ were maternally imprinted. In each scenario, we generated a range of POEs - $\mathrm{m} 1$ having $75 \%$ of the effect as compared to $\mathrm{p} 1, \mathrm{~m} 1$ having $50 \%$ of the effect as compared to $\mathrm{p} 1, \mathrm{~m} 1$ having $25 \%$ of the effect as compared to $\mathrm{p} 1$ and $\mathrm{m} 1$ having no effect on the phenotype. The first three conditions in each scenario represented partial maternal imprinting whereas the last condition in each scenario represented complete maternal imprinting. Using our approach $(\mathrm{H}-$ GCTA), we estimated the total fetal $\hat{\mathrm{h}}^{2}\left(\hat{\mathrm{h}}_{\mathrm{m} 1}^{2}+\hat{\mathrm{h}}_{\mathrm{p} 1}^{2}\right)$ as expected ( 50\%) (Fig 3, Supplementary Table 6). Results from H-GCTA showed that the variance attributable to $\mathrm{m} 1\left(\hat{\mathrm{h}}_{\mathrm{m} 1}^{2}\right)$ decreased whereas the variance attributable to $\mathrm{p} 1\left(\hat{\mathrm{h}}_{\mathrm{p} 1}^{2}\right)$ increased in accordance to the level of imprinting in each scenario. For example, in case of all variants with complete maternal imprinting $(\mathrm{m} 1 / \mathrm{p} 1=$ 0.0/1.0), variance attributable to $\mathrm{m} 1$ and $\mathrm{p} 1$ were $1.6 \%$ and $47.9 \%$ respectively (Supplementary Table 6). We also compared results from our approach with those from GCTA and M-GCTA. GCTA underestimated variance attributable to $\mathrm{f}\left(\hat{\mathrm{h}}_{\mathrm{f}}^{2}\right)$ depending on the proportion of causal variants with POEs and level of POEs. M-GCTA estimated variance attributable to $\mathrm{G}\left(\mathrm{h}_{\mathrm{g}}^{2}\right)$ was as expected $(\sim 50 \%)$ in case of partial imprinting; however, it underestimated the variance attributable to $\mathrm{G}$ in case of complete imprinting. (Supplementary Table 6). We further compared estimated variance based on $\mathrm{m} 1, \mathrm{~m} 2$ and $\mathrm{p} 1$ to calculate $\hat{\mathrm{h}}^{2}$ likely attributable to POEs (Supplementary Table 7).

\section{Heritability estimation of pregnancy phenotypes using empirical data}

All analyses for $\hat{\mathrm{h}}^{2}$ estimation were performed using a common set of $\sim 11$ million markers across $10,375$ mother-child pairs. In addition, two MAF cut-offs (0.001 and 0.01$)$ yielding approximately 9 million and 7 million markers respectively, were used for analysis. Only independent motherchild pairs (kinship coefficient < 0.05) were used in analysis and 20 principal components $(\mathrm{PCs})$ 
were used along with genotype-based GRMs in LMM (Supplementary Figure 3). For haplotypebased GRMs, we used 30 PCs (10 PCs corresponding to each haplotype) in LMM (Supplementary Figure 3). We estimated $\widehat{\mathrm{h}}^{2}$ using two methods, REML and HE regression. Using both methods, we also compared results from our approach (H-GCTA) with existing approaches, GCTA and MGCTA. Here, we describe results based on GRMs calculated through SNPs with MAF > 0.001. Results based on GRMs calculated through all polymorphic SNPs and SNPs with MAF $>0.01$ are provided in supplementary text, supplementary table 8 and 9.

\section{Heritability of gestational duration}

Using REML, the conventional GCTA approach estimated $\hat{\mathrm{h}}^{2}$ of gestational duration based on $\mathrm{m}$ and $\mathrm{f}-\left(\hat{\mathrm{h}}_{\mathrm{m}}^{2}=25.5 \%\right.$; S.E. $=4.8 \%$; $\mathrm{p}$ value $\left.=2.25 \mathrm{E}-08\right)$ and $\left(\hat{\mathrm{h}}_{\mathrm{f}}^{2}=9.5 \%\right.$; .E. $=4.5 \% ; \mathrm{p}$ value $=$ 1.62E-02). Our approach (H-GCTA) further resolved the heritability estimates based on $\mathrm{m} 1$ $14.0 \%($ S.E. $=4.6 \% ; \mathrm{p}$ value $=8.34 \mathrm{E}-04), \mathrm{m} 2-10.0 \%($ S.E. $=4.6 \% ; \mathrm{p}$ value $=1.33 \mathrm{E}-02)$ and $\mathrm{p} 1$ $-2.6 \%$ (S.E. $=4.4 \%$; p value $=2.77 \mathrm{E}-01)($ Fig $4 \mathrm{a})$. Using HE-regression, GCTA and H-GCTA generated similar results as through REML (Fig 4a). Results using our approach suggested that $\hat{\mathrm{h}}^{2}$ of gestational duration was primarily determined by maternal genome. Comparison with M-GCTA confirmed the results from H-GCTA and suggested that $\hat{\mathrm{h}}^{2}$ of gestational duration was mainly attributable to the SNPs which influence gestational duration through maternal genetic effect (Table 1a).

\section{Heritability of gestational duration adjusted birth weight}

Analysis using conventional GCTA showed that the estimated $\hat{\mathrm{h}}^{2}$ of birth weight based on $\mathrm{m}$ and f were $15.8 \%($ S.E. $=5.3 \%$; p value $=1.71 \mathrm{E}-03)$ and $32.3 \%($ S.E. $=5.4 \% ; \mathrm{p}$ value $=1.02 \mathrm{E}-09)$ respectively. Using our approach, we further distinguished phenotypic variance into variance components based on $\mathrm{m} 1-19.9 \%$ (S.E. $=5.3 \%$; $\mathrm{p}$ value $=9.27 \mathrm{E}-05) ; \mathrm{m} 2-3.0 \%($ S.E. $=4.9 \% ; \mathrm{p}$ value $=2.70 \mathrm{E}-01)$ and $\mathrm{p} 1-13.3 \%($ S.E. $=5.2 \% ; \mathrm{p}$ value $=5.03 \mathrm{E}-03)\left(\right.$ Fig 4b). The $\hat{\mathrm{h}}^{2}$ estimate obtained through H-GCTA suggested that narrow sense heritability of birth weight was primarily determined by the fetal genome. Comparison of $\hat{\mathrm{h}}^{2}$ estimates from our approach with those from M-GCTA illustrated that $\hat{\mathrm{h}}^{2}$ of birth weight was mainly attributable to the SNPs which influence birth weight only through direct effect (fetal effect) (Table 1b).

\section{Heritability of gestational duration adjusted birth length}

We estimated $\hat{\mathrm{h}}^{2}$ of birth length based on $\mathrm{m}\left(\hat{\mathrm{h}}_{\mathrm{m}}^{2}=21.8 \% ;\right.$ S.E. $=7.4 \% ; \mathrm{p}$ value $\left.=1.43 \mathrm{E}-03\right)$ and $\mathrm{f}\left(\widehat{\mathrm{h}}_{\mathrm{f}}^{2}=26.5 \%\right.$; S.E. $=7.5 \%$; $\mathrm{p}$ value $\left.=1.61 \mathrm{E}-04\right)$ using conventional GCTA approach. We further resolved the heritability estimates based on $\mathrm{m} 1-24.6 \%$ (S.E. $=7.4 \%$; $\mathrm{p}$ value $=3.45 \mathrm{E}-04) ; \mathrm{m} 2-$ $2.0 \%$ (S.E. $=7.1 \% ; \mathrm{p}$ value $=3.88 \mathrm{E}-01)$ and $\mathrm{p} 1-4.0 \%($ S.E. $=7.2 \% ; \mathrm{p}$ value $=2.85 \mathrm{E}-01)$ using our haplotype-based GCTA approach (Fig 4c). H-GCTA showed that unlike birth weight, variance in birth length was mainly attributable to $\mathrm{m} 1$ with a much smaller attribution to $\mathrm{p} 1$. In addition, the difference between $\left(\hat{\mathrm{h}}_{\mathrm{m} 1}^{2}-\widehat{\mathrm{h}}_{\mathrm{m} 2}^{2}\right)$ and $\widehat{\mathrm{h}}_{\mathrm{p} 1}^{2}\left(\widehat{\mathrm{h}}_{\mathrm{m} 1}^{2}-\widehat{\mathrm{h}}_{\mathrm{m} 2}^{2}-\widehat{\mathrm{h}}_{\mathrm{p} 1}^{2}=18.5 \%\right.$; S.E. $=12.5 \%$; $\mathrm{p}$ value $=6.88 \mathrm{E}-02$ ) suggested possible POE or correlation between maternal and fetal effects of the 
maternal transmitted alleles (Supplementary Table 10). As compared to the results from our approach, M-GCTA showed that variance in birth length was mainly attributable to the D i.e. SNPs with both indirect effect (maternal genetic effect) and direct effect (fetal effect) (d) (Table 1c).

\section{Heritability of gestational duration adjusted head circumference}

Narrow sense heritability $\left(\hat{\mathrm{h}}^{2}\right)$ of head circumference estimated using a conventional GCTA approach was $32.2 \%$ (S.E. $=9.0 \%$; p value $=1.45 \mathrm{E}-04)$ and $40.3 \%$ (S.E. $=9.2 \%$; p value $=5.50 \mathrm{E}-$ 06) based on $\mathrm{m}$ and $\mathrm{f}$ respectively. Using $\mathrm{H}-\mathrm{GCTA}$, we resolved the variance attributable to maternal and fetal genomes into heritability estimates based on $\mathrm{m} 1-33.1 \%$ (S.E. $=9.1 \%$; $\mathrm{p}$ value $=1.27 \mathrm{E}-04) ; \mathrm{m} 2-6.0 \%(\mathrm{~S} . \mathrm{E} .=8.7 \% ; \mathrm{p}$ value $=2.42 \mathrm{E}-01)$ and $\mathrm{p} 1-12.3 \%($ S.E. $=8.8 \% ; \mathrm{p}$ value $=7.85 \mathrm{E}-02)($ Fig $4 \mathrm{~d})$. Results from H-GCTA showed that the estimated $\hat{\mathrm{h}}^{2}$ of head circumference was mainly attributable to $\mathrm{m} 1 \mathrm{with}$ comparatively less attribution to $\mathrm{p} 1$. The results from M-GCTA analysis showed approximately equal contribution to variance of head circumference from $\mathrm{G}$ and D (Table 1d). The comparison of results from H-GCTA and M-GCTA suggested that head circumference was primarily determined by fetal genome with some influence through maternal genetic effect with a possible trend of POE or correlation between maternal and fetal effects of the maternal transmitted alleles (Supplementary Table 10). 


\section{Discussion}

Adverse pregnancy outcomes such as short gestational duration (preterm birth, affecting approximately $10 \%$ of all pregnancies), fetal growth restriction or macrosomia pose not only immediate but also long-term health risks to mother and child. Several studies have shown associations of preterm birth and low birth weight with developmental disabilities such as cerebral palsy, intellectual disabilities, autism spectrum disorder, attention deficit hyperactivity disorder, learning disability and future risk of type 2 diabetes mellitus and cardiovascular diseases in child ${ }^{40-}$ 45. Understanding the genetic architecture of pregnancy phenotypes will not only elucidate the genetic basis of immediate health outcomes but also shed light on the nature of their relationships with long-term health outcomes ${ }^{46}$.

To date, various genetic association and heritability estimation studies have attempted to reveal the genetic basis of gestational duration and fetal growth measures at birth in humans $21,23,28,30,41,47$ 51 . While genetic associations based on candidate genes or genome-wide markers reveal genotypephenotype relationships, heritability estimation using related (families) or unrelated samples provides quantitative measures of genetic contributions to phenotypic variance in a population. Heritability estimation based on epidemiological studies suggest that fetal genetic factors contribute $11-35 \%$ and $27-42 \%$ of variation in gestational duration and fetal growth measures at birth respectively whereas maternal genetic factors contribute $13-20 \%$ and $19-22 \%$ of variation in gestational duration and fetal growth measures at birth ${ }^{52-58}$ respectively. Similar patterns of maternal and fetal genetic contributions to the variance of pregnancy phenotypes are observed through genetic studies using GRMs based on an individual's genotypes ${ }^{21,23,27,28,30,41,48,59,60}$.

Pregnancy phenotypes are primarily genetically determined by two genomes - the maternal and fetal genome. These two genomes are correlated with each other through maternal transmitted alleles, sharing effect through both the mother and fetus. Therefore, most of the individual's genotype-based approaches for heritability estimation are limited in estimating explicit indirect (maternal effect) and direct (fetal effect) genetic contribution to pregnancy phenotypes. Using our haplotype-based GCTA approach (H-GCTA), we disentangle the contribution of maternal transmitted alleles (m1 - through maternal and fetal effect), maternal non-transmitted alleles (m2 - through maternal effect) and paternal transmitted alleles (p1 - through fetal effect) to the variance of gestational duration and gestational duration adjusted fetal growth measures at birth in 10,375 European mother-child pairs. Moreover, our approach can be extended to parent-child trios to detect the paternal genetic effect (genetic nurturing effect) ${ }^{22}$ (see results using simulated data).

Our results based on common and rare variants (SNPs with MAF > 0.001) show that approximately $14 \%$ and $10 \%$ variance in gestational duration is attributable to the $\mathrm{m} 1$ and $\mathrm{m} 2$ components respectively with a minimal contribution from p1 (Fig 4; Table 1). In contrast, variance in gestational duration adjusted fetal growth measures at birth are mainly contributed by $\mathrm{m} 1\left(\hat{\mathrm{h}}_{\mathrm{m} 1}^{2}=\right.$ $20-33 \%$ ) and p1 components $\left(\hat{\mathrm{h}}_{\mathrm{p} 1}^{2}=4-13 \%\right)$ with a minimal contribution from $\mathrm{m} 2$ (Fig 4; Table 1). Among fetal growth measures at birth, variance in birth weight has significant contributions from 
$\mathrm{m} 1\left(\hat{\mathrm{h}}_{\mathrm{m} 1}^{2}=20 \%\right)$ as well as $\mathrm{p} 1\left(\hat{\mathrm{h}}_{\mathrm{p} 1}^{2}=13 \%\right)$ whereas variance in birth length and head circumference are mainly driven by $\mathrm{m} 1$ (birth length: $\hat{\mathrm{h}}_{\mathrm{m} 1}^{2}=25 \%$; head circumference: $\hat{\mathrm{h}}_{\mathrm{m} 1}^{2}=$ $33 \%$ ). These new results suggest that variance in gestational duration is mainly driven by the mothers' genome whereas variance in fetal growth measures at birth is mainly driven by the fetal genome through direct fetal genetic effects. In addition, birth length and head circumference show evidence of parent-of-origin effects (POEs) based on the results from H-GCTA (Supplementary Table 10). Results using all polymorphic SNPs and SNPs with MAF > 0.01 support our findings based on SNPs with MAF > 0.001. We observed the largest $\hat{\mathrm{h}}^{2}$ estimates for each trait using all polymorphic SNPs, which decreased with increasing threshold of MAF cutoff (number of SNPs decrease with increasing MAF cutoff). Decrease in the $\hat{h}^{2}$ estimates with decrease in number of markers is a general limitation of GCTA-GREML which is dependent on several assumptions ${ }^{17,61}$.

Further, a comparison of results from our approach (H-GCTA) with those from M-GCTA confirms our observations regarding gestational duration and birth weight. While results from H-GCTA suggest major contribution to the variance of birth length and head circumference through $\mathrm{m} 1$, those from M-GCTA indicate primary attribution to direct-indirect effect covariance (D) i.e. SNPs showing effect through both the mother and fetus (Table 1). As suggested by previous results through M-GCTA ${ }^{23}$, birth length is jointly determined by maternal and fetal genomes. However, our current study with larger sample size suggests that H-GCTA or M-GCTA detect no major contribution through $\mathrm{m} 2$ or indirect effects (maternal genetic effect). In addition, a larger $\widehat{\mathrm{h}}_{\mathrm{m} 1}^{2}$ (contribution through maternal and fetal genetic effects) than the summation of $\hat{\mathrm{h}}_{\mathrm{m} 2}^{2}$ (contribution through maternal genetic effect) and $\hat{h}_{\mathrm{p} 1}^{2}$ (contribution through fetal genetic effect) suggests that birth length is mainly influenced by fetal alleles inherited from the maternal side. These observations suggest possible POEs or correlation between maternal and fetal effects in birth length and head circumference.

Interestingly, we observe that the contribution of $\mathrm{m} 1$ is larger than $\mathrm{m} 2$ or $\mathrm{p} 1$ for every pregnancy phenotype in the current study. There are several possible explanations for this pattern of results. The most obvious explanation is that $\mathrm{m} 1$ can influence a pregnancy phenotype through both the mother and fetus. For example, for a trait mainly defined by the maternal genome like gestational duration, higher contribution of $\mathrm{m} 1$ in comparison to $\mathrm{m} 2$ could be due to small but non-zero fetal effect of the $\mathrm{m} 1$ alleles (Supplementary Table 5). Similarly, for traits mainly defined by the fetal genome such as fetal size measurements at birth, higher contribution of $\mathrm{m} 1$ in comparison to $\mathrm{p} 1$ could be due to maternal effect of the $\mathrm{m} 1$ alleles (Supplementary Table 5). Assuming maternalfetal additivity (maternal effect and fetal effect are defined by independent sets of variants and no interaction between maternal and fetal effect) and no POE, $\left(\hat{\mathrm{h}}_{\mathrm{m} 1}^{2}-\hat{\mathrm{h}}_{\mathrm{m} 2}^{2}\right)$ is equal to $\hat{\mathrm{h}}_{\mathrm{p} 1}^{2}$ (Supplementary Table 5). Due to possible POE, above equality doesn't hold for birth length and head circumference (Supplementary Table 10). The above pattern can also be observed due to correlations or interactions of indirect maternal and direct fetal effects. Besides the abovementioned explanations, several other biological phenomena such as interaction between SNPs 
within the mother or fetus (epistasis), gene-environment interaction and transmission distortion can explain the observed pattern of $\widehat{h}^{2}$ for gestational duration and fetal size measurements at birth.

Despite the above advances, our current approach has limitations. First, heritability estimation in our approach can be affected if assumptions such as the normal distribution of effect sizes, LD between assayed variants and causal variants, absence of epistasis (gene-gene interaction), absence of gene-environment interaction and the absence of non-additive effects are not met. This set of limitations is shared with all current GCTA-based approaches. Second, splitting the phenotypic variance attributable to $\mathrm{m} 1\left(\hat{\mathrm{h}}_{\mathrm{m} 1}^{2}\right)$ into fractions contributed through indirect effect (maternal genetic effect) and direct (fetal) effect isn't upfront. Although, the proportion of $\hat{\mathrm{h}}_{\mathrm{m} 1}^{2}$ contributed through indirect effects (maternal genetic effect) and direct (fetal) effect can be calculated as $\left(\hat{\mathrm{h}}_{\mathrm{m} 1}^{2}\right.$ - $\left.\hat{\mathrm{h}}_{\mathrm{p} 1}^{2}\right)$ and $\left(\hat{\mathrm{h}}_{\mathrm{m} 1}^{2}-\hat{\mathrm{h}}_{\mathrm{m} 2}^{2}\right)$ respectively. These calculations are based on two major assumptions maternal-fetal additivity (independent sets of SNPs influencing pregnancy phenotypes through mother and fetus and there is no interaction among them) and no POE. Third, our hypotheses of a higher contribution of $\mathrm{m} 1$ to the $\hat{\mathrm{h}}^{2}$ of pregnancy phenotypes needs further support from larger datasets and simulations depicting interactions of indirect maternal and direct fetal effects in the presence and absence of POE.

In conclusion, our approach can resolve the phenotypic variance into indirect effects (maternal genetic effect) and direct (fetal) effect by considering mother/child pair as a single analytical unit with three distinct haplotypes - $\mathrm{m} 1, \mathrm{~m} 2$ and $\mathrm{p} 1$. In comparison to M-GCTA, our approach can further disentangle the variance attributable to direct (fetal) effect into maternal and fetal components through $\mathrm{m} 1$ and $\mathrm{p} 1$, which enables assessment of possible POEs or correlation between maternal and fetal effects. Moreover, our approach can be extended to study parental effects in duos/trios data ${ }^{22}$. We believe this approach represents a significant enhance to the genetic analytic toolbox of pregnancy that others will also employ moving forward. 


\section{References}

1. Visscher, P.M., Hill, W.G. \& Wray, N.R. Heritability in the genomics era--concepts and misconceptions. Nat Rev Genet 9, 255-66 (2008).

2. Zhang, Z. et al. Mixed linear model approach adapted for genome-wide association studies. Nat Genet 42, 355-60 (2010).

3. Cnaan, A., Laird, N.M. \& Slasor, P. Using the general linear mixed model to analyse unbalanced repeated measures and longitudinal data. Stat Med 16, 2349-80 (1997).

4. Henderson, C.R. Applications of Linear Models in Animal Breeding, 462 (University of Guelph, 1984).

5. Vinkhuyzen, A.A., Wray, N.R., Yang, J., Goddard, M.E. \& Visscher, P.M. Estimation and partition of heritability in human populations using whole-genome analysis methods. Annu Rev Genet 47, 7595 (2013).

6. Thompson, H.D.P.a.R. Recovery of Inter-Block Information when Block Sizes are Unequal. Biometrika 58, 545-554 (1971).

7. Lee, S.H. \& van der Werf, J.H. An efficient variance component approach implementing an average information REML suitable for combined LD and linkage mapping with a general complex pedigree. Genet Sel Evol 38, 25-43 (2006).

8. Chen, J., Zhang, D. \& Davidian, M. A Monte Carlo EM algorithm for generalized linear mixed models with flexible random effects distribution. Biostatistics 3, 347-60 (2002).

9. Fong, Y., Rue, H. \& Wakefield, J. Bayesian inference for generalized linear mixed models. Biostatistics 11, 397-412 (2010).

10. Stahl, E.A. et al. Bayesian inference analyses of the polygenic architecture of rheumatoid arthritis. Nat Genet 44, 483-9 (2012).

11. Yang, J., Lee, S.H., Goddard, M.E. \& Visscher, P.M. GCTA: a tool for genome-wide complex trait analysis. Am J Hum Genet 88, 76-82 (2011).

12. Yang, J. et al. Common SNPs explain a large proportion of the heritability for human height. Nat Genet 42, 565-9 (2010).

13. Speed, D., Hemani, G., Johnson, M.R. \& Balding, D.J. Improved heritability estimation from genome-wide SNPs. Am J Hum Genet 91, 1011-21 (2012).

14. Zaitlen, N. et al. Using extended genealogy to estimate components of heritability for 23 quantitative and dichotomous traits. PLoS Genet 9, e1003520 (2013).

15. Bulik-Sullivan, B.K. et al. LD Score regression distinguishes confounding from polygenicity in genome-wide association studies. Nat Genet 47, 291-5 (2015).

16. Golan, D., Lander, E.S. \& Rosset, S. Measuring missing heritability: inferring the contribution of common variants. Proc Natl Acad Sci U S A 111, E5272-81 (2014).

17. Yang, J., Zeng, J., Goddard, M.E., Wray, N.R. \& Visscher, P.M. Concepts, estimation and interpretation of SNP-based heritability. Nat Genet 49, 1304-1310 (2017).

18. Yang, J. et al. Genome partitioning of genetic variation for complex traits using common SNPs. Nat Genet 43, 519-25 (2011).

19. Yang, J. et al. Genetic variance estimation with imputed variants finds negligible missing heritability for human height and body mass index. Nat Genet 47, 1114-20 (2015).

20. Speed, D. et al. Reevaluation of SNP heritability in complex human traits. Nat Genet 49, 986-992 (2017).

21. Warrington, N.M., Freathy, R.M., Neale, M.C. \& Evans, D.M. Using structural equation modelling to jointly estimate maternal and fetal effects on birthweight in the UK Biobank. Int J Epidemiol 47, 1229-1241 (2018).

22. Kong, A. et al. The nature of nurture: Effects of parental genotypes. Science 359, 424-428 (2018). 
23. Eaves, L.J., Pourcain, B.S., Smith, G.D., York, T.P. \& Evans, D.M. Resolving the effects of maternal and child genotype on dyadic outcomes in genome wide complex trait analysis ("M-GCTA"). Behav Genet 44, 445-55 (2014).

24. Zhang, G. et al. Genetic studies of gestational duration and preterm birth. Best Pract Res Clin Obstet Gynaecol 52, 33-47 (2018).

25. Wolf, J.B. \& Wade, M.J. What are maternal effects (and what are they not)? Philos Trans $R$ Soc Lond B Biol Sci 364, 1107-15 (2009).

26. Doolin, M.T. et al. Maternal genetic effects, exerted by genes involved in homocysteine remethylation, influence the risk of spina bifida. Am J Hum Genet 71, 1222-6 (2002).

27. Beaumont, R.N. et al. Genome-wide association study of child birth weight in 86577 women identifies five novel loci and highlights maternal genetic effects that are independent of fetal genetics. Hum Mol Genet 27, 742-756 (2018).

28. Qiao, Z. et al. Introducing M-GCTA a Software Package to Estimate Maternal (or Paternal) Genetic Effects on Child Phenotypes. Behav Genet (2019).

29. Zhang, G. et al. Assessing the Causal Relationship of Maternal Height on Birth Size and Gestational Age at Birth: A Mendelian Randomization Analysis. PLoS Med 12, e1001865 (2015).

30. Zhang, G. et al. Genetic Associations with Gestational Duration and Spontaneous Preterm Birth. N Engl J Med 377, 1156-1167 (2017).

31. Sham, P.C. \& Purcell, S. Equivalence between Haseman-Elston and variance-components linkage analyses for sib pairs. Am J Hum Genet 68, 1527-32 (2001).

32. Xu, X., Weiss, S., Xu, X. \& Wei, L.J. A unified Haseman-Elston method for testing linkage with quantitative traits. Am J Hum Genet 67, 1025-8 (2000).

33. Haseman, J.K. \& Elston, R.C. The investigation of linkage between a quantitative trait and a marker locus. Behav Genet 2, 3-19 (1972).

34. Fraser, A. et al. Cohort Profile: the Avon Longitudinal Study of Parents and Children: ALSPAC mothers cohort. Int J Epidemiol 42, 97-110 (2013).

35. Boyd, A. et al. Cohort Profile: the 'children of the 90s'--the index child of the Avon Longitudinal Study of Parents and Children. Int J Epidemiol 42, 111-27 (2013).

36. Olsen, J. et al. The Danish National Birth Cohort--its background, structure and aim. Scand J Public Health 29, 300-7 (2001).

37. Magnus, P. et al. Cohort Profile Update: The Norwegian Mother and Child Cohort Study (MoBa). Int J Epidemiol 45, 382-8 (2016).

38. Group, H.S.C.R. The Hyperglycemia and Adverse Pregnancy Outcome (HAPO) Study. Int J Gynaecol Obstet 78, 69-77 (2002).

39. Plunkett, J. et al. An evolutionary genomic approach to identify genes involved in human birth timing. PLoS Genet 7, e1001365 (2011).

40. Schieve, L.A. et al. Population impact of preterm birth and low birth weight on developmental disabilities in US children. Ann Epidemiol 26, 267-74 (2016).

41. Warrington, N.M. et al. Maternal and fetal genetic effects on birth weight and their relevance to cardio-metabolic risk factors. Nat Genet 51, 804-814 (2019).

42. Jornayvaz, F.R. et al. Low birth weight leads to obesity, diabetes and increased leptin levels in adults: the CoLaus study. Cardiovasc Diabetol 15, 73 (2016).

43. Harder, T., Rodekamp, E., Schellong, K., Dudenhausen, J.W. \& Plagemann, A. Birth weight and subsequent risk of type 2 diabetes: a meta-analysis. Am J Epidemiol 165, 849-57 (2007).

44. Barker, D.J. The developmental origins of chronic adult disease. Acta Paediatr Supp/ 93, 26-33 (2004).

45. James-Todd, T.M. et al. Gestational age, infant birth weight, and subsequent risk of type 2 diabetes in mothers: Nurses' Health Study II. Prev Chronic Dis 10, E156 (2013). 
46. Chen, J. et al. Haplotype genetic score analysis in 10,734 mother/infant pairs reveals complex maternal and fetal genetic effects underlying the associations between maternal phenotypes, birth outcomes and adult phenotypes. bioRxiv, 737106 (2019).

47. Laurin, C. et al. Partitioning Phenotypic Variance Due to Parent-of-Origin Effects Using Genomic Relatedness Matrices. Behav Genet 48, 67-79 (2018).

48. Horikoshi, M. et al. Genome-wide associations for birth weight and correlations with adult disease. Nature 538, 248-252 (2016).

49. Bezold, K.Y., Karjalainen, M.K., Hallman, M., Teramo, K. \& Muglia, L.J. The genomics of preterm birth: from animal models to human studies. Genome Med 5, 34 (2013).

50. Muglia, L.J. \& Katz, M. The enigma of spontaneous preterm birth. N Engl J Med 362, 529-35 (2010).

51. Plunkett, J. \& Muglia, L.J. Genetic contributions to preterm birth: implications from epidemiological and genetic association studies. Ann Med 40, 167-95 (2008).

52. Wu, W. et al. The heritability of gestational age in a two-million member cohort: implications for spontaneous preterm birth. Hum Genet 134, 803-8 (2015).

53. York, T.P., Eaves, L.J., Neale, M.C. \& Strauss, J.F., 3rd. The contribution of genetic and environmental factors to the duration of pregnancy. Am J Obstet Gynecol 210, 398-405 (2014).

54. York, T.P. et al. Fetal and maternal genes' influence on gestational age in a quantitative genetic analysis of 244,000 Swedish births. Am J Epidemiol 178, 543-50 (2013).

55. Mook-Kanamori, D.O. et al. Heritability estimates of body size in fetal life and early childhood. PLoS One 7, e39901 (2012).

56. Kistka, Z.A. et al. Heritability of parturition timing: an extended twin design analysis. Am J Obstet Gynecol 199, 43 e1-5 (2008).

57. Lunde, A., Melve, K.K., Gjessing, H.K., Skjaerven, R. \& Irgens, L.M. Genetic and environmental influences on birth weight, birth length, head circumference, and gestational age by use of population-based parent-child data. Am J Epidemiol 165, 734-41 (2007).

58. Clausson, B., Lichtenstein, P. \& Cnattingius, S. Genetic influence on birthweight and gestational length determined by studies in child of twins. BJOG 107, 375-81 (2000).

59. Liu, X. et al. Variants in the fetal genome near pro-inflammatory cytokine genes on $2 \mathrm{q} 13$ associate with gestational duration. Nat Commun 10, 3927 (2019).

60. van der Valk, R.J. et al. A novel common variant in DCST2 is associated with length in early life and height in adulthood. Hum Mol Genet 24, 1155-68 (2015).

61. Wray, N.R. et al. Pitfalls of predicting complex traits from SNPs. Nat Rev Genet 14, 507-15 (2013).

62. Chang, C.C. et al. Second-generation PLINK: rising to the challenge of larger and richer datasets. Gigascience 4, 7 (2015).

63. Delaneau, O., Marchini, J. \& Zagury, J.F. A linear complexity phasing method for thousands of genomes. Nat Methods 9, 179-81 (2011).

64. Durbin, R. Efficient haplotype matching and storage using the positional Burrows-Wheeler transform (PBWT). Bioinformatics 30, 1266-72 (2014).

65. McCarthy, S. et al. A reference panel of 64,976 haplotypes for genotype imputation. Nat Genet 48, 1279-83 (2016). 


\section{Acknowledgement}

We are extremely grateful to all the families who participated in Avon Longitudinal Study of Parents And Children (ALSPAC), Hyperglycemia and Adverse Pregnancy Outcome study (HAPO), Finnish Birth Cohort (FIN), Danish Birth Cohort (DNBC) and Norwegian Mother, Father and Child Cohort study (MoBa), the clinical staff for their consistent help, the whole team of respective studies including interviewers, computer and laboratory technicians, clerical workers, research scientists, volunteers, managers, receptionists and nurses. We also thank organizing bodies for administrating the studies. Our sincere thanks to $\mathrm{dbGaP}$ for depositing and hosting data access for the current research. 


\section{Funding}

This work is supported by a grant from the Burroughs Wellcome Fund (10172896), the March of Dimes Prematurity Research Center Ohio Collaborative, a grant from the Bill and Melinda Gates Foundation (OPP1175128), and grants from the Cincinnati Children's Hospital Medical Center (GAP/RIP).

The Norwegian Mother, Father and Child Cohort Study is supported by the Norwegian Ministry of Health and Care Services and the Ministry of Education and Research. We are grateful to all the participating families in Norway who take part in this on-going cohort study. We thank the Norwegian Institute of Public Health (NIPH) for generating high-quality genomic data. This research is part of the HARVEST collaboration, supported by the Research Council of Norway (\#229624). We also thank the NORMENT Centre for providing genotype data, funded by the Research Council of Norway (\#223273), South East Norway Health Authority and KG Jebsen Stiftelsen. We further thank the Center for Diabetes Research, the University of Bergen for providing genotype data and performing quality control and imputation of the data funded by the ERC AdG project SELECTionPREDISPOSED, Stiftelsen Kristian Gerhard Jebsen, Trond Mohn Foundation, the Research Council of Norway, the Novo Nordisk Foundation, the University of Bergen, and the Western Norway health Authorities (Helse Vest).

The genotyping and analyses were supported by the grants from: Jane and Dan Olsson Foundations (Gothenburg, Sweden), Swedish Medical Research Council (2015-02559), Norwegian Research Council/FUGE (grant no. 151918/S10; FRI-MEDBIO 249779), March of Dimes (21-FY16-121), and the Burroughs Wellcome Fund Preterm Birth Research Grant (10172896) and by Swedish government grants to researchers in the public health sector (ALFGBG-717501, ALFGBG507701, ALFGBG-426411).

The UK Medical Research Council and Wellcome (Grant ref: 102215/2/13/2) and the University of Bristol provide core support for ALSPAC. GWAS data was generated by Sample Logistics and Genotyping Facilities at Wellcome Sanger Institute and LabCorp (Laboratory Corporation of America) using support from 23andMe. A comprehensive list of grants funding is available on the ALSPAC Website (http://www.bristol.ac.uk/alspac/external/documents/grantacknowledgements.pdf).

The DNBC datasets used for the analyses described in this manuscript were obtained from dbGaP at http://www.ncbi.nlm.nih.gov/sites/entrez?db=gap through dbGaP accession number phs000103.v1.p1. The GWAS of Prematurity and its Complications study is one of the genomewide association studies funded as part of the Gene Environment Association Studies (GENEVA) under the Genes, Environment and Health Initiative (GEI).

The HAPO datasets used for the analyses described in this manuscript were obtained from dbGaP at http://www.ncbi.nlm.nih.gov/sites/entrez?db=gap through $\mathrm{dbGaP}$ accession number phs000096.v4.p1. This study is part of the Gene Environment Association Studies initiative (GENEVA) funded by the trans-NIH Genes, Environment, and Health Initiative (GEI). 


\section{Figures and Tables}

\section{a) Schematic Difference between genotype and haplotype-based analysis approach}

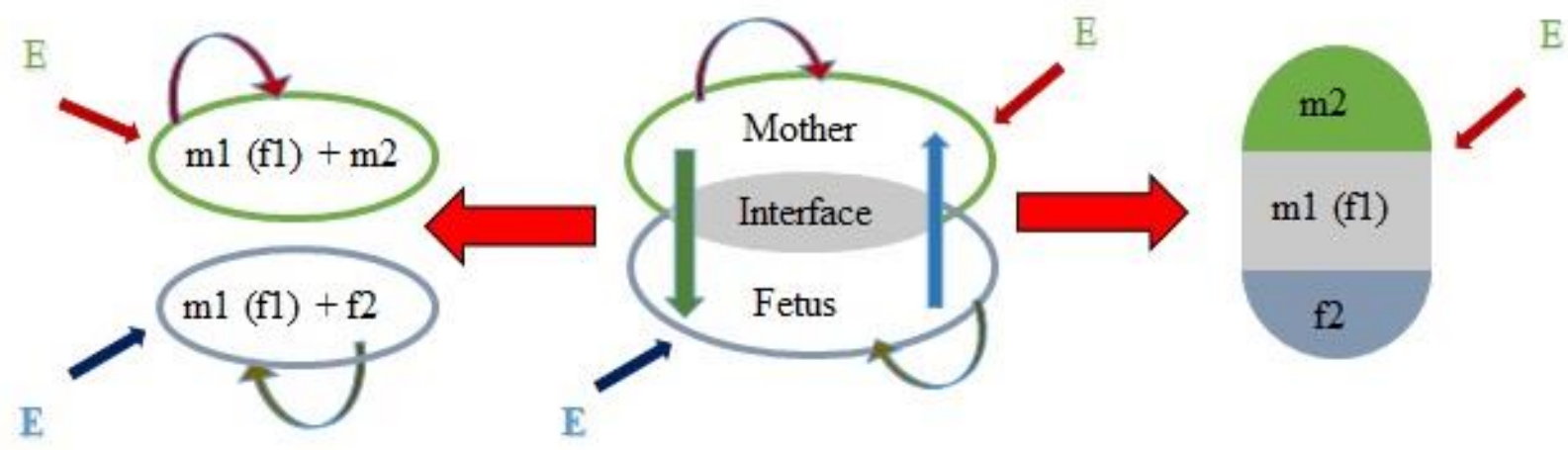

b)

Genotype-based REML

Haplotype-based REML

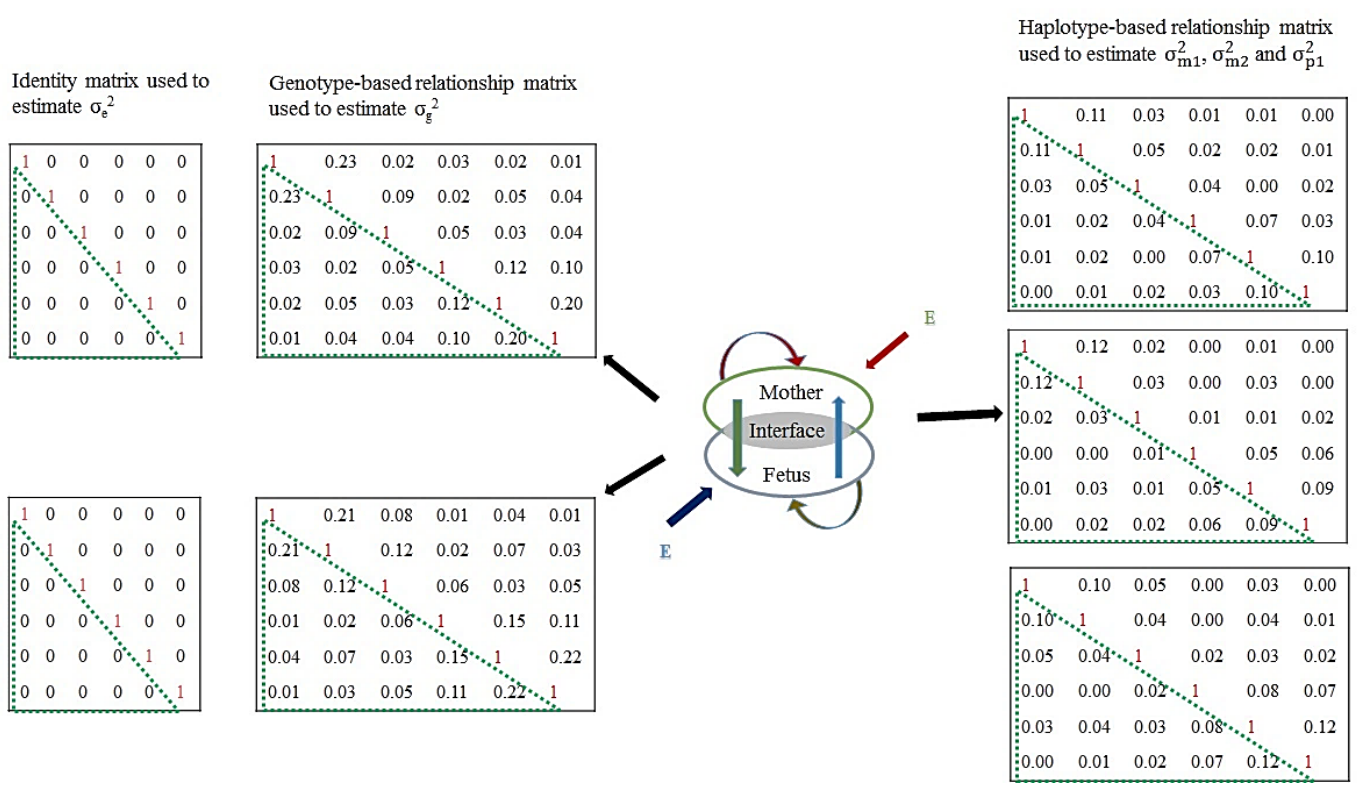

Fig 1: a) Schematic representation of the difference between the genotype and haplotype-based analysis approach; the left part of the figure represents the conventional approach based on genotypes of mothers and child separately and the right part represents haplotype-based analysis for pregnancy phenotypes by treating mother/child pairs as analytical units. b) Schematic representation of the difference between conventional approach of heritability estimation utilizing genotype-based GRMs and our approach utilizing haplotype-based GRMs (representing the example of mother-child duos). m1 (f1): Maternal transmitted alleles; m2: Maternal nontransmitted alleles; f2 (p1): paternal transmitted alleles; E: Environmental factors; $\sigma_{\mathrm{g}}^{2}$ : phenotypic variance attributable to mothers' or children's genotypes; $\sigma_{\mathrm{m} 1}^{2}, \sigma_{\mathrm{m} 2}^{2}$ and $\sigma_{\mathrm{p} 1}^{2}$ : phenotypic variance attributable to $\mathrm{m} 1, \mathrm{~m} 2$ and $\mathrm{p} 1$ respectively; $\sigma_{\mathrm{e}}^{2}$ : phenotypic variance attributable to $\mathrm{E}$. 
a)

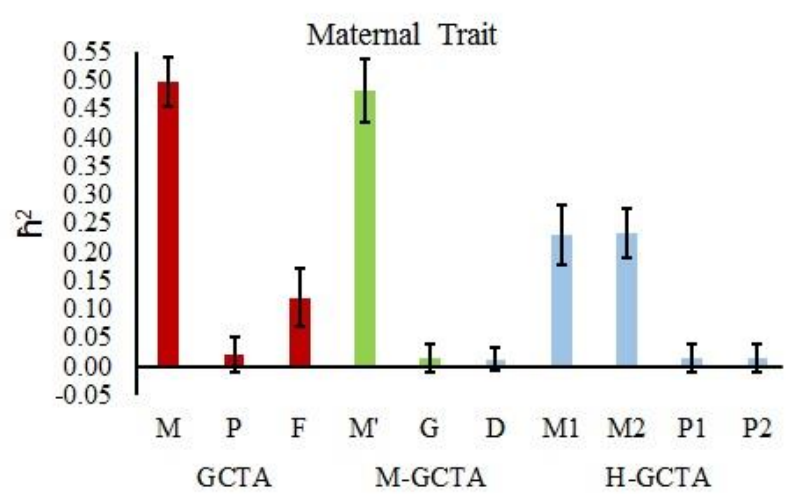

b)

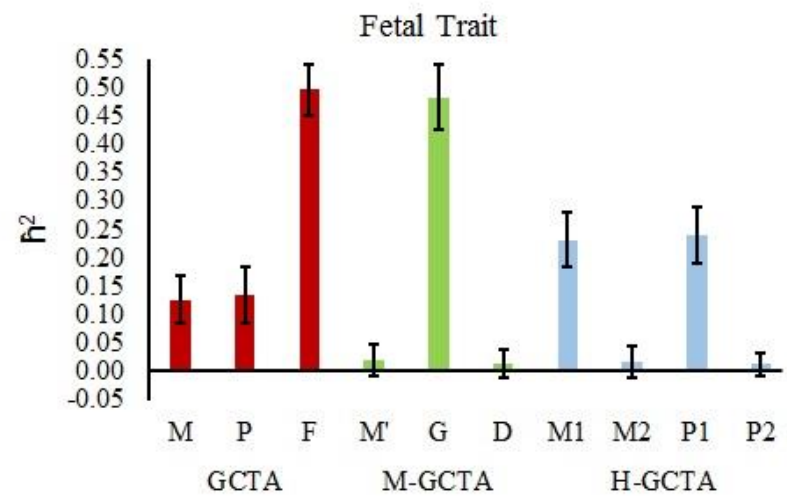

Fig 2: $\hat{\mathrm{h}}^{2}$ estimates using GCTA, M-GCTA and H-GCTA in simulated genotypes and phenotypes data; a) $\hat{h}^{2}$ estimates for maternal traits i.e. phenotypes have genetic contribution only through mother; b) $\hat{h}^{2}$ estimates for fetal traits i.e. phenotypes are genetic contribution only through fetus. For GCTA, M is the GRM generated from maternal genotypes ( $\mathrm{m}), \mathrm{P}$ is the GRM generated from paternal genotypes (p), and F is the GRM generated from fetal genotypes (f). For M-GCTA, M' represents the genetic relationship matrix of mothers; $G$ represents genetic relationship matrix of children and D represents mother-child covariance matrix. For H-GCTA, M1 is the GRM generated from maternal transmitted alleles (m1), M2 is the GRM generated from maternal nontransmitted alleles (m2), P1 is the GRM generated from paternal transmitted alleles (p1), and P2 is the GRM generated from paternal non-transmitted alleles (p2). The calculation of these matrices and the estimation of phenotypic variance attributable to these components are described in the Methods section. 
a)

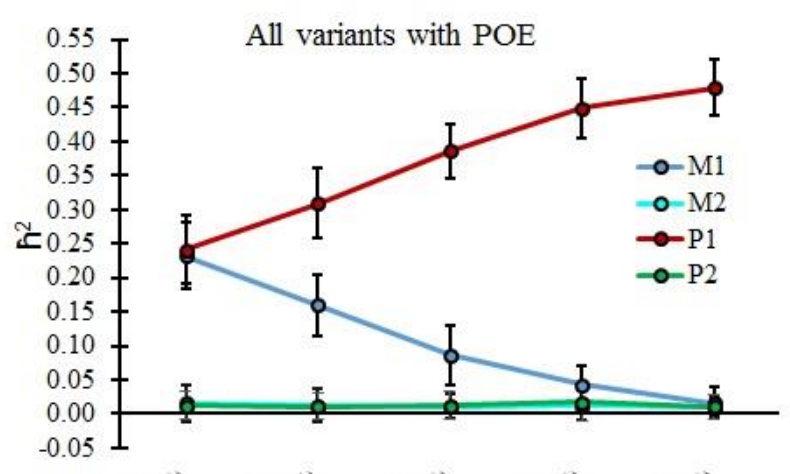

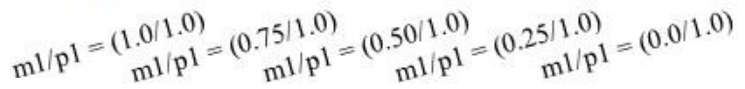

c)

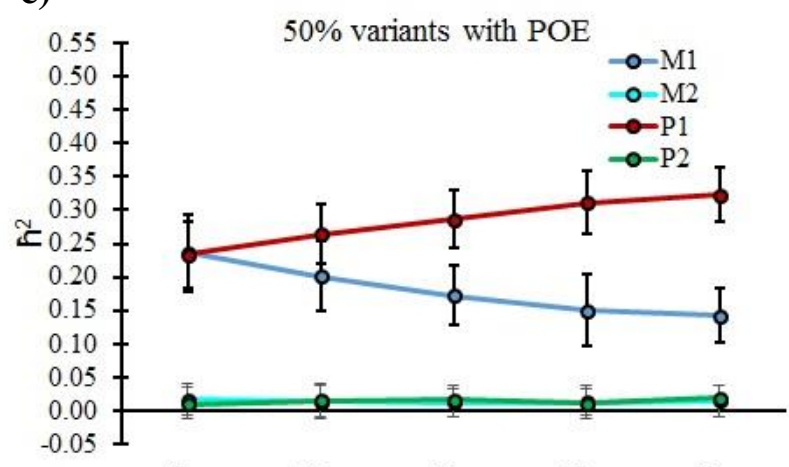

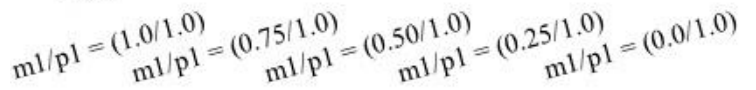

b)

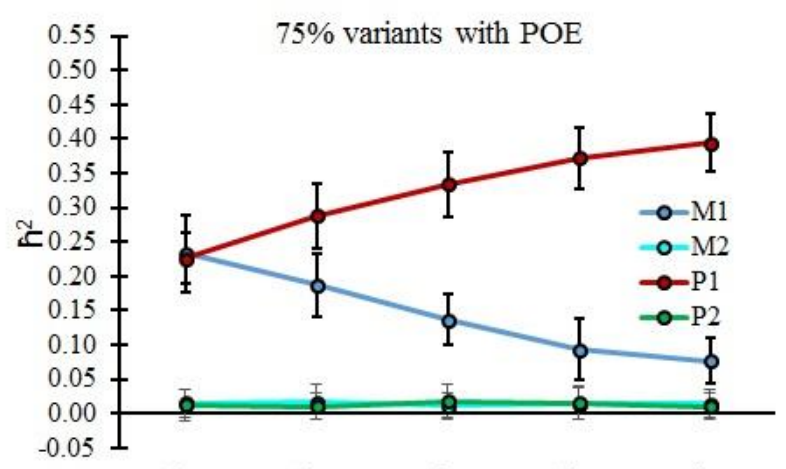

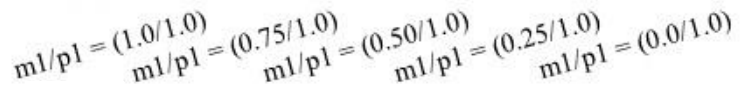

d)

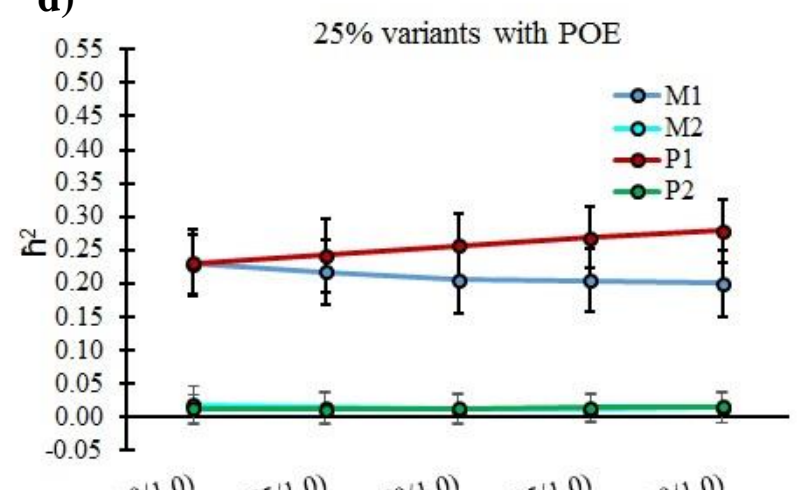

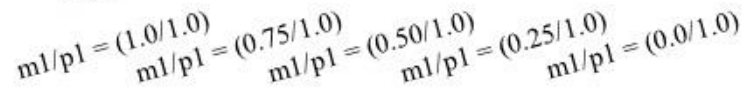

Fig 3: $\hat{\mathrm{h}}^{2}$ estimates using our approach (H-GCTA) in simulated fetal traits: a) All variants show parent-of-origin effects (POEs); b) 75\% variants show POEs; c) 50\% variants show POEs; d) 25\% variants show POEs. In each scenario, maternal transmitted alleles (m1) show either no imprinting - effect of $\mathrm{m} 1$ is equal to effect of paternal transmitted alleles (p1) or partial imprinting - effect size of $\mathrm{m} 1$ is $0.75-0.25$ in comparison to $\mathrm{p} 1$ or complete imprinting - $\mathrm{m} 1$ has no effect on phenotype. 
a) SNP heritability estimates of gestational duration

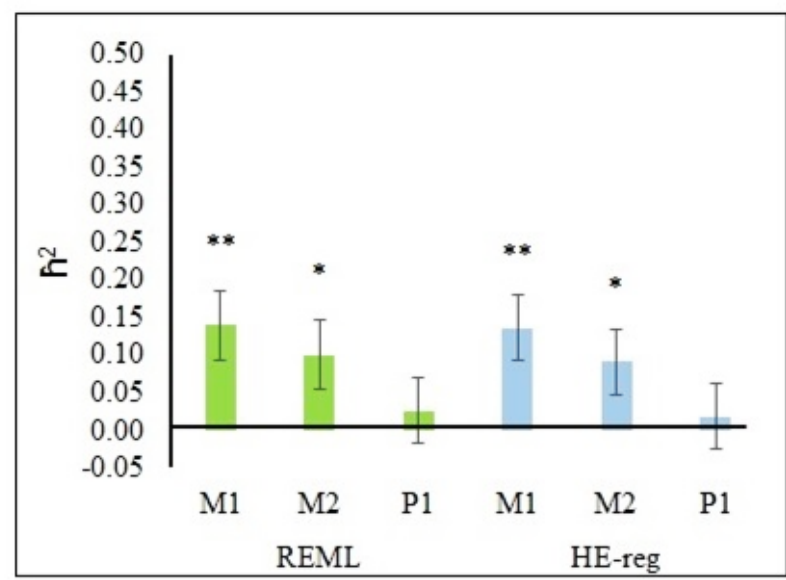

c) SNP heritability estimates of birth length

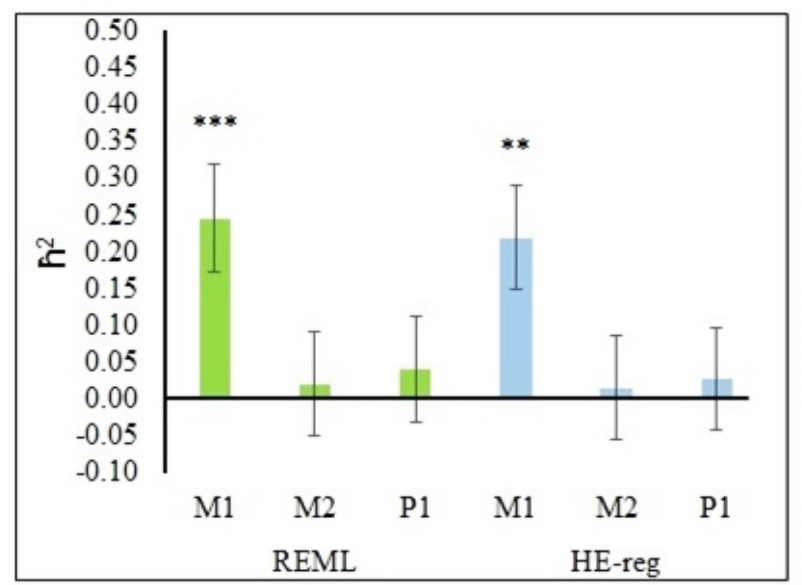

b) SNP heritability estimates of birth weight

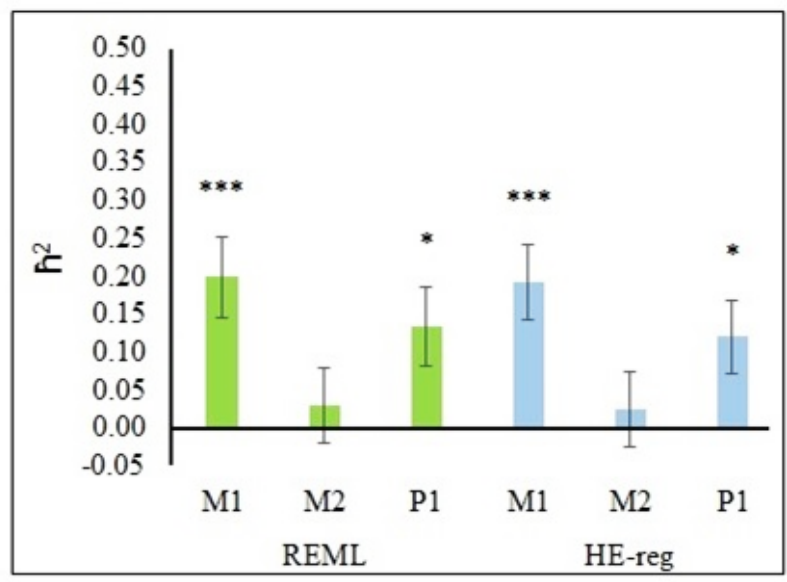

d) SNP heritability estimates of head circumference

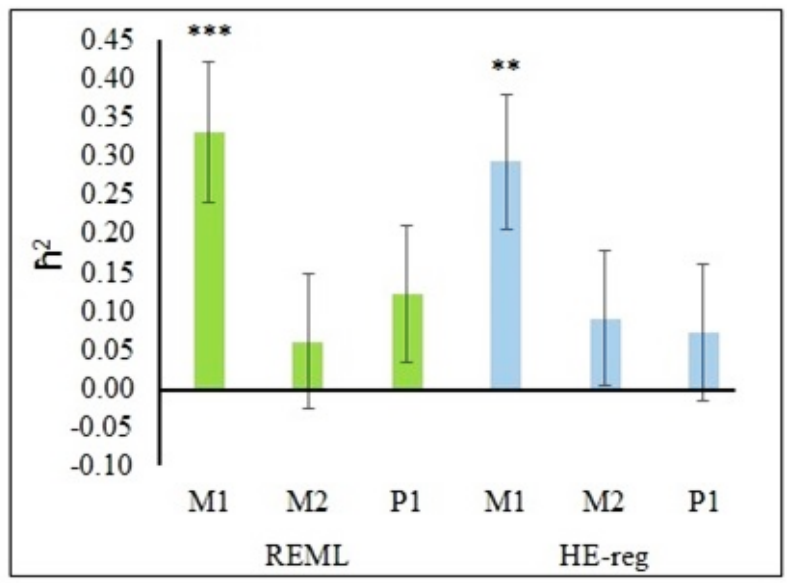

Fig 4: $\hat{\mathrm{h}}^{2}$ estimates of pregnancy phenotypes using our approach (H-GCTA), implementing REML and $\mathrm{HE}$ regression in unrelated mother-child pairs (relatedness cutoff >0.05). M1: GRM generated from maternal transmitted alleles (m1); M2: GRM generated from maternal non-transmitted alleles (m2); P1: GRM generated from paternal transmitted alleles (p1). All analyses were adjusted for 30 principal components (PCs). 
a) $\hat{\mathrm{h}}^{2}$ estimates of gestational duration

\begin{tabular}{|ccc|c|c|c|c|c|}
\hline Approach & GRM & \multicolumn{4}{c|}{ REML } & \multicolumn{3}{c|}{ HE-Reg } \\
\hline & & $\hat{\mathbf{h}}^{\mathbf{2}}$ & S.E. & p-val & $\hat{\mathbf{h}}^{2}$ & S.E. & p-val \\
GCTA & M & 0.2546 & 0.0475 & $2.25 \mathrm{E}-08$ & 0.2317 & 0.0433 & $8.40 \mathrm{E}-08$ \\
& $\mathrm{~F}$ & 0.0952 & 0.0455 & $1.62 \mathrm{E}-02$ & 0.0905 & 0.0433 & $3.68 \mathrm{E}-02$ \\
\hline \multirow{3}{*}{ M-GCTA } & M' & 0.2287 & 0.0617 & $8.37 \mathrm{E}-05$ & 0.2049 & 0.0567 & $2.99 \mathrm{E}-04$ \\
& $\mathrm{G}$ & 0.0349 & 0.0590 & $2.76 \mathrm{E}-01$ & 0.0279 & 0.0573 & $6.26 \mathrm{E}-01$ \\
\hline \multirow{3}{*}{ H-GCTA } & D & 0.0079 & 0.0478 & $4.35 \mathrm{E}-01$ & 0.0171 & 0.0452 & $7.06 \mathrm{E}-01$ \\
\hline & M1 & 0.1397 & 0.0458 & $8.34 \mathrm{E}-04$ & 0.1356 & 0.0434 & $1.76 \mathrm{E}-03$ \\
& P1 & 0.1005 & 0.0458 & $1.33 \mathrm{E}-02$ & 0.0908 & 0.0434 & $3.65 \mathrm{E}-02$ \\
\hline
\end{tabular}

b) $\hat{h}^{2}$ estimates of birth weight

\begin{tabular}{|cccccccc|}
\hline Approach & GRM & \multicolumn{3}{c|}{ REML } & \multicolumn{3}{c|}{ HE-Reg } \\
& & $\hat{\mathbf{h}}^{\mathbf{2}}$ & S.E. & p-val & $\hat{\mathbf{h}}^{\mathbf{2}}$ & S.E. & p-val \\
GCTA & M & 0.1579 & 0.0533 & $1.71 \mathrm{E}-03$ & 0.1334 & 0.0491 & $6.62 \mathrm{E}-03$ \\
& $\mathrm{~F}$ & 0.3226 & 0.0543 & $1.02 \mathrm{E}-09$ & 0.2895 & 0.0492 & $4.09 \mathrm{E}-09$ \\
\hline \multirow{3}{*}{ M-GCTA } & M' & 0.0570 & 0.0652 & $1.88 \mathrm{E}-01$ & 0.0387 & 0.0643 & $5.48 \mathrm{E}-01$ \\
& G & 0.2619 & 0.0692 & $5.48 \mathrm{E}-05$ & 0.2332 & 0.0650 & $3.33 \mathrm{E}-04$ \\
& D & 0.0488 & 0.0524 & $1.78 \mathrm{E}-01$ & 0.0404 & 0.0513 & $4.31 \mathrm{E}-01$ \\
\hline \multirow{3}{*}{ H-GCTA } & M1 & 0.1985 & 0.0533 & $9.27 \mathrm{E}-05$ & 0.1911 & 0.0493 & $1.04 \mathrm{E}-04$ \\
& M2 & 0.0300 & 0.0495 & $2.70 \mathrm{E}-01$ & 0.0241 & 0.0493 & $6.24 \mathrm{E}-01$ \\
& P1 & 0.1329 & 0.0520 & $5.03 \mathrm{E}-03$ & 0.1197 & 0.0493 & $1.52 \mathrm{E}-02$ \\
\hline
\end{tabular}

c) $\hat{\mathrm{h}}^{2}$ estimates for Birth Length

\begin{tabular}{|cccccccc|}
\hline Approach & GRM & \multicolumn{3}{c|}{ REML } & \multicolumn{3}{c|}{ HE-Reg } \\
\hline & & $\hat{\mathbf{h}}^{\mathbf{2}}$ & S.E. & p-val & $\hat{\mathbf{h}}^{\mathbf{2}}$ & S.E. & p-val \\
\hline \multirow{2}{*}{ GCTA } & M & 0.2175 & 0.0741 & $1.43 \mathrm{E}-03$ & 0.2012 & 0.0695 & $3.80 \mathrm{E}-03$ \\
& F & 0.2646 & 0.0748 & $1.61 \mathrm{E}-04$ & 0.2459 & 0.0697 & $4.18 \mathrm{E}-04$ \\
\hline \multirow{3}{*}{ M-GCTA } & M' & 0.0000 & 0.0929 & $5.00 \mathrm{E}-01$ & -0.0214 & 0.0906 & $8.13 \mathrm{E}-01$ \\
& G & 0.0442 & 0.0939 & $2.84 \mathrm{E}-01$ & 0.0351 & 0.0920 & $7.03 \mathrm{E}-01$ \\
& D & 0.2065 & 0.0716 & $2.09 \mathrm{E}-03$ & 0.1987 & 0.0727 & $6.27 \mathrm{E}-03$ \\
\hline \multirow{3}{*}{ H-GCTA } & M1 & 0.2455 & 0.0740 & $3.45 \mathrm{E}-04$ & 0.2192 & 0.0698 & $1.69 \mathrm{E}-03$ \\
& M2 & 0.0198 & 0.0706 & $3.88 \mathrm{E}-01$ & 0.0148 & 0.0699 & $8.33 \mathrm{E}-01$ \\
& P1 & 0.0404 & 0.0715 & $2.85 \mathrm{E}-01$ & 0.0268 & 0.0699 & $7.02 \mathrm{E}-01$ \\
\hline
\end{tabular}




\section{d) $\widehat{h}^{2}$ estimates for Head Circumference}

\begin{tabular}{|cccccccc|}
\hline Approach & GRM & \multicolumn{3}{c|}{ REML } & \multicolumn{3}{c|}{ HE-Reg } \\
\hline & & $\hat{\mathbf{h}}^{\mathbf{2}}$ & S.E. & p-val & $\hat{\mathbf{h}}^{\mathbf{2}}$ & S.E. & p-val \\
\hline \multirow{2}{*}{ GCTA } & M & 0.3221 & 0.0904 & $1.45 \mathrm{E}-04$ & 0.3128 & 0.0876 & $3.58 \mathrm{E}-04$ \\
& $\mathrm{~F}$ & 0.4030 & 0.0924 & $5.50 \mathrm{E}-06$ & 0.3535 & 0.0878 & $5.70 \mathrm{E}-05$ \\
\hline \multirow{3}{*}{ M-GCTA } & M' & 0.0590 & 0.1087 & $2.87 \mathrm{E}-01$ & 0.0618 & 0.1149 & $5.91 \mathrm{E}-01$ \\
& G & 0.1711 & 0.1154 & $6.54 \mathrm{E}-02$ & 0.0987 & 0.1158 & $3.94 \mathrm{E}-01$ \\
& $\mathrm{D}$ & 0.2332 & 0.0856 & $3.96 \mathrm{E}-03$ & 0.2361 & 0.0912 & $9.58 \mathrm{E}-03$ \\
\hline \multirow{3}{*}{ H-GCTA } & M1 & 0.3310 & 0.0914 & $1.27 \mathrm{E}-04$ & 0.2928 & 0.0878 & $8.51 \mathrm{E}-04$ \\
& M2 & 0.0603 & 0.0870 & $2.42 \mathrm{E}-01$ & 0.0906 & 0.0878 & $3.02 \mathrm{E}-01$ \\
& P1 & 0.1233 & 0.0882 & $7.85 \mathrm{E}-02$ & 0.0729 & 0.0878 & $4.06 \mathrm{E}-01$ \\
\hline
\end{tabular}

Table 1: $\hat{\mathrm{h}}^{2}$ estimates in unrelated mother-child pairs (relatedness cutoff > 0.05) using REML and HE-regression through genotype-based GCTA approach, M-GCTA and haplotype-based GCTA (H-GCTA) approach for a) gestational duration and b) birth weight c) birth length and d) head circumference. GRMs were generated using SNPs with MAF > 0.001. Gestational duration was adjusted for fetal sex and fetal growth measures at birth were additionally adjusted for gestational duration up to third orthogonal polynomial. Analyses using GCTA and M-GCTA approach were adjusted for 20 PCs and H-GCTA approach was adjusted for 30 PCs (10 PCs corresponding to $\mathrm{m} 1, \mathrm{~m} 2$ and $\mathrm{p} 1$ each). 


\section{Methods}

\section{Datasets and quality control}

We used genome wide single nucleotide polymorphism (SNP) data from 10,375 mother-child pairs from five European cohorts to estimate $\hat{\mathrm{h}}^{2}$ of gestational duration and fetal growth measures at birth (birth weight, birth length and head circumference). These included three data sets collected from Nordic countries - Danish Birth Cohort (DNBC), Finnish dataset (FIN) and Norwegian Mother, Father and child Cohort study (MoBa) for genetic study of preterm birth, Avon Longitudinal Study of Parents and Children (ALSPAC) from UK, and Hyperglycemia and Adverse Pregnancy Outcome study (HAPO) from UK, Canada, and Australia. (Supplementary Tables 1-4, Supplementary Figure 2). A detailed description of data sets can be found in Supplementary Text.

Genotyping of DNA extracted from whole blood or swab samples was done on various SNP array platforms such as Affymetrix 6.0, Illumina Human550-Quad, Illumina Human610-Quad, Illumina Human 660W-Quad. SNP array data was filtered based on SNP and sample quality. Quality Control (QC) of genotypes data was performed at two levels - marker level and individual level. Marker level QC was conducted using PLINK $1.9^{62}$ on the basis of SNP call rate, minor allele frequency (MAF), Hardy-Weinberg Equilibrium (HWE) and individual level QC was done on the basis of call rate per individual, average heterozygosity per individual, sex assignment, inbreeding coefficient. Non-European samples were removed from the study by principal components analysis (PCA) anchored with 1,000 genome samples. Following QC, genotype data of motherchild pairs were phased using SHAPEIT $2^{63}$. SHAPEIT 2 automatically recognizes pedigree information provided in the input files. When phasing mother/child duos together, the first allele in child was always the transmitted allele from mother and the second one from father. We imputed the pre-phased genotypes for missing genotypes on Sanger Imputation Server using Positional Burrows-Wheeler Transform (PBWT) software ${ }^{64}$. Haplotype reference consortium (HRC) panel was utilized as reference data for imputation purpose ${ }^{65}$. The phasing and mother-child allele transmission of the imputed alleles were retained from the pre-phasing stage.

QC of phenotype data was conducted considering gestational duration as the primary outcome. Pregnancies involving history of risk factors for preterm birth or any medical complication during pregnancy influencing preterm birth, $\mathrm{C}$-sections and non-spontaneous births were excluded. We also excluded, non-singlet pregnancies, pregnancies who self-reported non-European ancestry and children who could not survive $>1$ year. Additionally, gestational duration was adjusted for fetal sex; fetal growth measures at birth such as birth weight, birth length and head circumference were adjusted for gestational duration up to third orthogonal polynomial component. Details of genotype and phenotype QC is provided in the Supplementary Text.

\section{Statistical Method}

We used a linear mixed model (LMM) to estimate the SNP heritability $\left(\hat{\mathrm{h}}^{2}\right)$ of pregnancy phenotypes. This model assumes that the phenotype was normally distributed $-\mathrm{Y} \sim \mathrm{N}(\mu, \mathrm{V})$ with mean $\mu$ and variance V. We created GRMs from standardized genotypes/haplotypes utilizing the method developed by Yang et.al., 2010 ${ }^{11,12}$. Each cell of the genotype-based GRM and haplotype- 
based GRM represented relatedness between two individuals $\mathrm{j}$ and $\mathrm{k}$ calculated based on genotypes (Equation 1) and haplotypes (Equation 2) respectively.

$A_{j k}=\frac{1}{w} \sum_{i=1}^{w} \frac{\left(x_{i j}-2 p_{i}\right)\left(x_{i k}-2 p_{i}\right)}{2 p_{i}\left(1-p_{i}\right)}$

(Equation 1)

Where, $\mathrm{A}_{\mathrm{jk}}$ is the correlation coefficient between two individuals $\mathrm{j}$ and $\mathrm{k}$ averaged over all SNPs; $\mathrm{w}$ is number of SNPs used to calculate relatedness; $\mathrm{x}_{\mathrm{ij}}$ is the number of copies of the reference alleles in individual $\mathrm{j}$ for SNP $\mathrm{i}$ (i.e. 0 or 1 or 2 ); $\mathrm{x}_{\mathrm{ik}}$ is the number of copies of the reference alleles in individual $\mathrm{k}$ for SNP $\mathrm{i}$ (0 or 1 or 2$)$; $\mathrm{p}_{\mathrm{i}}$ is frequency of reference allele of SNP $\mathrm{i}$.

$\mathrm{T}_{\mathrm{jk}}=\frac{1}{\mathrm{w}} \sum_{\mathrm{i}=1}^{\mathrm{w}} \frac{\left(\mathrm{c}_{\mathrm{ij}}-\mathrm{p}_{\mathrm{i}}\right)\left(\mathrm{c}_{\mathrm{ik}}-\mathrm{p}_{\mathrm{i}}\right)}{\mathrm{p}_{\mathrm{i}}\left(1-\mathrm{p}_{\mathrm{i}}\right)}$

(Equation 2)

Where, $\mathrm{T}_{\mathrm{jk}}$ is the correlation coefficient between two mother/child duos or full trios $\mathrm{j}$ and $\mathrm{k}$ based on maternal transmitted alleles $(\mathrm{m} 1)$ or maternal non-transmitted alleles (m2) or paternal transmitted alleles ( $\mathrm{p} 1$ ) or paternal non-transmitted alleles (p2); $w$ is number of SNPs whose alleles are used to calculate relatedness; $c_{i j}$ is the number of the reference alleles of $m 1$ or $m 2$ or $p 1$ or $\mathrm{p} 2$ in mother/child duo or full trio $\mathrm{j}$ for SNP i (i.e. 0 or 1); $\mathrm{c}_{\mathrm{ik}}$ is the number of the reference alleles of $\mathrm{m} 1$ or $\mathrm{m} 2$ or $\mathrm{p} 1$ or $\mathrm{p} 2$ in mother/child duo or full trio $\mathrm{k}$ for SNP i (i.e. 0 or 1); $\mathrm{p}_{\mathrm{i}}$ is frequency of reference allele of SNP i.

For genotypes-based analysis, we created two GRMs - M and F by utilizing maternal genotypes (m) and fetal genotypes (f) respectively. For haplotypes-based analysis, we considered motherchild pair as a single analytical unit consisting of three haplotypes corresponding to $\mathrm{m} 1, \mathrm{~m} 2$, and p1. We created three separate GRMs - M1, M2 and P1 using only m1, only m2 and only p1 respectively (Fig 1a, b). We fitted mothers' genotype-based GRM (M) (Equations 3 and 4) and children's genotype-based GRM (F) (Equations 5 and 6) separately in LMM to estimate phenotypic variance attributable to maternal and fetal genotypes respectively. To calculate explicit contribution of maternal and fetal genomes to the overall narrow-sense heritability of pregnancy phenotypes, we simultaneously fitted all three matrices (M1, M2 and P1) in LMM and estimated the additive genetic variance attributable to each of three components (Equation 7, 8).

$\mathrm{Y}_{\mathrm{s}}=\mathrm{X} \beta+\mathrm{Z}_{\mathrm{m}} \mathrm{u}_{\mathrm{m}}+\epsilon$

$\mathrm{Y}_{\mathrm{s}} \mathrm{Y}_{\mathrm{s}}^{\prime}=\mathrm{XX} \mathrm{X}^{\prime} \sigma_{\beta}^{2}+\mathrm{M} \sigma_{\mathrm{M}}^{2}+\mathrm{I} \sigma_{\epsilon}^{2}$

$\mathrm{Y}_{\mathrm{s}}=\mathrm{X} \beta+\mathrm{Z}_{\mathrm{f}} \mathrm{u}_{\mathrm{f}}+\epsilon$

$\mathrm{Y}_{\mathrm{s}} \mathrm{Y}_{\mathrm{s}}^{\prime}=\mathrm{XX} \mathrm{X}^{\prime} \sigma_{\beta}^{2}+\mathrm{F} \sigma_{\mathrm{F}}^{2}+\mathrm{I} \sigma_{\epsilon}^{2}$

$\mathrm{Y}_{\mathrm{s}}=\mathrm{X} \beta+\mathrm{Z}_{\mathrm{m} 1} \mathrm{u}_{\mathrm{m} 1}+\mathrm{Z}_{\mathrm{m} 2} \mathrm{u}_{\mathrm{m} 2}+\mathrm{Z}_{\mathrm{p} 1} \mathrm{u}_{\mathrm{p} 1}+\epsilon$

$\mathrm{Y}_{\mathrm{s}} \mathrm{Y}_{\mathrm{s}}^{\prime}=\mathrm{XX} \mathrm{X}^{\prime} \sigma_{\beta}^{2}+\mathrm{M} 1 \sigma_{\mathrm{M} 1}^{2}+\mathrm{M} 2 \sigma_{\mathrm{M} 2}^{2}+\mathrm{P} 1 \sigma_{\mathrm{P} 1}^{2}+\mathrm{I} \sigma_{\epsilon}^{2}$

Where, $Y_{s}$ is a vector of standardized phenotype ( $\mathrm{n}$ 1; where, $\mathrm{n}$ is number of individuals); $\mathrm{X}$ is a matrix of covariates representing fixed effects ( $\mathrm{n} x \mathrm{p}$; where, $\mathrm{p}$ is number of fixed effects); $\beta$ is a vector of fixed effects ( $\mathrm{p} \times 1) ; \mathrm{Z}_{\mathrm{m}}$ is a matrix of mothers' standardized genotypes $(\mathrm{m})(\mathrm{n} \times \mathrm{w}$; where, $w$ is number of SNPs); $Z_{f}$ is a matrix of children's standardized genotypes (f) ( $\left.\mathrm{nx} \mathrm{w}\right) ; \mathrm{Z}_{\mathrm{m} 1}$ is a matrix of standardized maternal transmitted alleles $(\mathrm{m} 1)(\mathrm{n} \times \mathrm{w}) ; \mathrm{Z}_{\mathrm{m} 2}$ is a matrix of 
standardized maternal non-transmitted alleles $(\mathrm{m} 2)(\mathrm{nx} \mathrm{w}) ; \mathrm{Z}_{\mathrm{p} 1}$ is a matrix of standardized paternal transmitted alleles (p1) ( $\mathrm{nxw}) ; \varepsilon$ is a vector of residual effects with $\varepsilon \sim \mathrm{N}\left(0, \mathrm{I} \sigma_{\varepsilon}^{2}\right) ; \mathrm{u}_{\mathrm{m}}$ and $\mathrm{u}_{\mathrm{f}}$ are vectors of random effect sizes for maternal genotypes $(\mathrm{m})$ and fetal genotypes (f); $\mathrm{u}_{\mathrm{m} 1}, \mathrm{u}_{\mathrm{m} 2}$ and $\mathrm{u}_{\mathrm{p} 1}$ are vectors of random effect sizes for maternal transmitted (m1), maternal non-transmitted (m2) and paternal transmitted $(\mathrm{p} 1)$ alleles respectively $(\mathrm{m} \times 1) ; \mathrm{Y}_{\mathrm{S}} \mathrm{Y}_{\mathrm{S}}^{\prime}$ is Variance-Covariance matrix of phenotypes; M, F, M1, M2 and P1 are GRMs generated from $Z_{m}, Z_{f}, Z_{m 1}, Z_{m 2}$ and $Z_{p 1}$ respectively (e.g. $M=Z_{m 1} Z_{m 1}^{\prime}$ ); $\sigma^{2}$ are the variances of the respective components.

Assuming additivity, the effect of the maternal transmitted alleles is the summation of the maternal effect $\left(u_{m 1}^{\prime}\right)$ and fetal effect $\left(u_{m 1}^{\prime \prime}\right)$ conferred by the same maternal transmitted alleles $\left(u_{m 1}=\right.$ $\left.u_{m 1}^{\prime}+u_{m 1}^{\prime \prime}\right)$. The maternal effect of the maternal transmitted alleles $\left(u_{m 1}^{\prime}\right)$ should be equal to the maternal effect of the maternal non-transmitted alleles $\left(u_{m 2}\right)$. Thus, the fetal effect of the maternal transmitted alleles can be expressed as $\mathrm{u}_{\mathrm{m} 1}^{\prime \prime}=\mathrm{u}_{\mathrm{m} 1}-\mathrm{u}_{\mathrm{m} 2}$. Therefore, we evaluated POE - the overall difference of the fetal effect of maternal and paternal transmitted allele $\left(u_{m 1}^{\prime \prime} v s . u_{p 1}\right)$ by testing $\left(\widehat{\mathrm{h}}_{\mathrm{m} 1}^{2}-\widehat{\mathrm{h}}_{\mathrm{m} 2}^{2}-\widehat{\mathrm{h}}_{\mathrm{p} 1}^{2}\right)=0$ using one sample $\mathrm{z}$ test (one-tailed). A pooled standard error (S.E.) was calculated using $\sqrt{\left(\mathrm{S} . \mathrm{E}_{\mathrm{m}_{\mathrm{m} 1}}\right)^{2}+\left(\mathrm{S} . \mathrm{E}_{\mathrm{m}_{2}}\right)^{2}+\left(\mathrm{S} . \mathrm{E}_{\cdot \mathrm{p} 1}\right)^{2}}$. Where, $\hat{\mathrm{h}}_{\mathrm{m} 1}^{2}, \hat{\mathrm{h}}_{\mathrm{m} 2}^{2}$ and $\hat{\mathrm{h}}_{\mathrm{p} 1}^{2}$ are variance components based on maternal transmitted alleles, maternal non-transmitted alleles and paternal transmitted alleles respectively; S.E.m1, S.E.m2 and S.E.p1 are standard errors corresponding to $\hat{\mathrm{h}}^{2}$ based on $\mathrm{m} 1, \mathrm{~m} 2$ and $\mathrm{p} 1$ respectively.

It should be noted that the above test of POE requires assumption of independence between maternal effects $\left(u_{m 1}^{\prime}\right)$ and fetal effects $\left(u_{m 1}^{\prime \prime}\right)$ of the maternal transmitted alleles. If there is correlation between $\mathrm{u}_{\mathrm{m} 1}^{\prime}$ and $\mathrm{u}_{\mathrm{m} 1}^{\prime \prime}\left(\rho=\operatorname{Corr}\left(\mathrm{u}_{\mathrm{m} 1}^{\prime}, \mathrm{u}_{\mathrm{m} 1}^{\prime \prime}\right) \neq 0\right), \mathrm{h}_{\mathrm{m} 1}^{2}=\mathrm{h}_{\mathrm{m} 1}^{2}+\mathrm{h}_{\mathrm{m} 1}^{2}{ }^{\prime \prime}+\mathrm{h}_{\mathrm{cov}}^{2}$, where $\mathrm{h}_{\mathrm{m} 1}^{2}{ }^{\prime}$ and $\mathrm{h}_{\mathrm{m} 1}^{2}{ }^{\prime \prime}$ are the additive genetic variance explained by the maternal and fetal effects of the maternal transmitted alleles. $\mathrm{h}_{\text {cov }}^{2}$ is the covariance between the maternal and fetal effects, which can be expressed as $\int q(1-q) \pi(q)\left(2 \mathrm{~s}_{\mathrm{m} 1}^{\prime} \mathrm{s}_{\mathrm{m} 1}^{\prime \prime} \rho\right) d q$, where $\mathrm{s}_{\mathrm{m} 1}^{\prime}$ and $\mathrm{s}_{\mathrm{m} 2}^{\prime \prime}$ are the standard deviation of $\mathrm{u}_{\mathrm{m} 1}^{\prime}$ and $\mathrm{u}_{\mathrm{m} 1}^{\prime \prime}$, respectively. $q$ and $\pi(q)$ are the allele frequency and the allele frequency spectrum. In this case, a non-zero $\left(\hat{\mathrm{h}}_{\mathrm{m} 1}^{2}-\widehat{\mathrm{h}}_{\mathrm{m} 2}^{2}-\widehat{\mathrm{h}}_{\mathrm{p} 1}^{2}\right)$ may suggest POE (i.e. $\left.\mathrm{u}_{\mathrm{m} 1}^{\prime \prime} \neq \mathrm{u}_{\mathrm{p} 1}\right)$ or correlation between maternal effect $\left(u_{m 1}^{\prime}\right)$ and fetal effect $\left(u_{m 1}^{\prime \prime}\right)$ of the maternal transmitted alleles.

\section{Implementation}

Phenotypic variance i.e. $\operatorname{Var}(\mathrm{Y})$ attributable to different components could be estimated by fitting GRMs corresponding to those components in LMM or regression model. We used REML and HE regression methods implemented in GCTA to estimate $\hat{\mathrm{h}}^{2}$. For genotype-based analysis through conventional GCTA approach, we fitted a GRM generated from mothers' genotypes (M) and children's genotypes (F) separately in LMM or HE-regression whereas for haplotype-based analysis through H-GCTA approach, we fitted three GRMs (M1, M2 and P1) simultaneously in LMM or HE-regression. We also compared results from our approach with those from M-GCTA. Analysis through the M-GCTA approach involved generation of the GRMs using mothers' and children's genotypes together. The upper left quadrant of the GRM represented genetic 
relationship matrix of mothers (M'); the lower right quadrant represented genetic relationship matrix of children $(\mathrm{G})$ and sum of the lower left quadrant and its transpose represented the genetic relationship matrix of mothers and children (D).

\section{Simulation}

We simulated 3,000 trios consisting of four sets of haplotypes (m1, m2, p1, p2) with 10,000 SNPs. Each SNP was simulated as random draw from Bernoulli distribution of minor allele frequency (MAF) and repeated 3,000 times for each haplotype. MAF information was picked from randomly chosen SNPs from chromosome 20 from 1,000 genomes project European samples data (EUR_AF $>0.001)$. Effect size corresponding to each SNP was simulated as random draw form a normal distribution $[\mathrm{N}(0,1)] . \widehat{\mathrm{h}}^{2}$ was assumed $50 \%$ and environmental components (e) were random draws from a normal distribution $\left[\mathrm{N}\left(0,\left(1-\hat{\mathrm{h}}^{2}\right)^{1 / 2}\right)\right]$. Three sets of phenotypes were simulated considering effects only from the mother (maternal traits), the father (paternal traits) or child (fetal traits). We also simulated fetal traits with POE, where $\mathrm{m} 1$ had less effect in comparison to $\mathrm{p} 1$. We considered four scenarios, where varying fractions of causal variants $(25 \%, 50 \%, 75 \%$ and 100\%) showed maternal imprinting. In each scenario, we simulated different levels of imprinting for $\mathrm{m} 1$ (25\% $100 \%)$ by reducing effect sizes of $\mathrm{m} 1(75 \%-0 \%)$ as compared to $\mathrm{p} 1$. Non-zero effects of $\mathrm{m} 1$ as compared to $\mathrm{p} 1$ represented partial maternal imprinting whereas no effect of $\mathrm{m} 1$ represented complete imprinting. All simulations were replicated one hundred times. All relatedness matrices using simulated data were generated and fitted into LMM in a similar way as mentioned in the statistical methods section.

\section{Analysis of Empirical datasets}

We performed analyses using three sets of markers - all polymorphic SNPs, SNPs with MAF > 0.001 and SNPs with MAF > 0.01, to include the contribution of very rare, rare and common variants to the heritability of pregnancy phenotypes (Supplementary Figure 1). The marker sets based on the MAF cutoff were selected in each dataset separately, considering mothers as founders. Then, a common set of markers across all datasets was selected in each MAF cutoff category. We pooled individual datasets and generated five different GRMs utilizing mothers' genotypes (M), children's genotypes (F), maternal transmitted haplotypes (M1), maternal non-transmitted haplotypes (M2) and paternal transmitted haplotypes (P1) using the imputed genotype data of mother/child pairs (Supplementary Table 2). One of the related individuals was removed from each GRM (relatedness coefficient > 0.05) and a common set of mother-child pairs across five GRMs was selected in each MAF cutoff category (Supplementary Table 3). We fitted these GRMs in LMM or HE regression model as described in implementation section. All the analyses were adjusted for principal components (PCs) - 20 PCs for analyses through GCTA and M-GCTA and 30 PCs (10 PCs corresponding to $\mathrm{m} 1, \mathrm{~m} 2$ and $\mathrm{p} 1$ each) for analyses through H-GCTA (Supplementary Figure 3). We also replicated our findings in another Nordic dataset of $~ 8,000$ mother-child pairs. We estimated the $\hat{\mathrm{h}}^{2}$ of gestational length through REML in replication dataset using SNPs with MAF $>0.01$. 